\title{
Detecting susceptibility genes for rheumatoid arthritis based on a novel sliding-window approach Qiuying Sha ${ }^{1}$, Rui Tang ${ }^{1}$ and Shuanglin Zhang* ${ }^{1,2}$
}

\author{
Addresses: ${ }^{1}$ Department of Mathematical Sciences, Michigan Technological University, 1400 Townsend Drive, Houghton, MI 49931, USA and \\ ${ }^{2}$ Mathematical Sciences, Heilongjiang University, Harbin 150080, PR China \\ E-mail: Qiuying Sha - qsha@mtu.edu; Rui Tang - rtang@mtu.edu; Shuanglin Zhang* - shuzhang@mtu.edu \\ *Corresponding author
}

from Genetic Analysis Workshop 16

St Louis, MO, USA 17-20 September 2009

Published: 15 December 2009

BMC Proceedings 2009, 3(SuppI 7):SI4 doi: 10.1 I86/I753-656I-3-S7-SI4

This article is available from: http://www.biomedcentral.com/I753-656//3/S7/SI4

(c) 2009 Sha et al; licensee BioMed Central Ltd.

This is an open access article distributed under the terms of the Creative Commons Attribution License (http://creativecommons.org/licenses/by/2.0), which permits unrestricted use, distribution, and reproduction in any medium, provided the original work is properly cited.

\begin{abstract}
With the recent rapid improvements in high-throughout genotyping techniques, researchers are facing a very challenging task of large-scale genetic association analysis, especially at the wholegenome level, without an optimal solution. In this study, we propose a new approach for genetic association analysis based on a variable-sized sliding-window framework. This approach employs principal component analysis to find the optimal window size. Using the bisection algorithm in window size searching, the proposed method tackles the exhaustive computation problem. It is more efficient and effective than currently available approaches. We conduct the genome-wide association study in Genetic Analysis Workshop I6 (GAWI6) Problem I data using the proposed method. Our method successfully identified several susceptibility genes that have been reported by other researchers and additional candidate genes for follow-up studies.
\end{abstract}

\section{Background}

With the availability of large-scale genotyping technologies, the cost of genome-wide analyses has been greatly reduced and a boom of large-scale genetic association studies is underway. A sliding-window approach, in which several neighboring single-nucleotide polymorphisms (SNPs) together included in a "window frame", is a popular strategy of multiple allelic association analysis. During the test the window slides across the genome region under study in a stepwise fashion [1-3]. Variable sized sliding-window approaches with variable window sizes decided by the underlying linkage disequilibrium (LD) pattern perform more efficiently in large-scale data analysis. The problem for variable sized sliding-window approaches is how to search the optimal window size with being not only computationally practical but also statistically sufficient to gain higher detection power for both common and rare risk factors.

In this report, based on the variable sized slidingwindow frame, we adapt the optimal window size to the local LD pattern by employing principal components (PC) approach. The PC approach is known as a linear projection method that defines a lower-dimensional space and captures the maximum information of the initial data [4]. Each optimal window size is defined by 
the first few PCs (i.e., 3 or 5) that could explain a main fraction of the total amount (i.e., $90 \%$ or $95 \%$ ) of information in the data.

\section{Data}

In our study, we used the Genetic Analysis Workshop (GAW) 16 Problem 1 data, which is the initial batch of the whole-genome association data for the North American Rheumatoid Arthritis Consortium (NARAC). Data were available for 868 cases and 1194 controls. There are 22 chromosomes with 545,080 SNP-genotype fields from the Illumina 550k chip. To avoid the missing value problem, any subject who had missing values in that window was excluded from the current window. Thus, some subjects may not be in the current window but will still be included in the study in other windows. In this way, we retained the most information we could.

\section{Methods}

\section{Optimal window size defined by PC analysis}

We consider a study with total $M$ individuals in a data set and with genotype information denoted by vectors $G_{i}=$ $\left(g_{i 1}, g_{i 2}, \ldots, g_{i N}\right)^{T}(i=1,2, \ldots, M)$ at N SNP loci for the $i^{\text {th }}$ individual. We code the genotype $g_{i j}$ as 0,1 , or 2 for the number of minor (less frequent) alleles at SNP $j, j=$ $1,2 \ldots, N$ of individual $i$. Let $y_{i}$ denote the trait value of individual $i$.

In the sliding-window frame, a window denoted as $w_{1}^{b}$ is a set of neighboring SNPs $\{b, b+1, b+2, \ldots, b+l-1\}$. A variable sized sliding window which begins with SNP $b$, denoted as $\Omega^{b}$, is a collection of windows $w_{l}^{b}$ with $l$ ranging from $s$ to $\Gamma^{b}$, where $s$ and $\Gamma^{b}$ are the smallest and largest window sizes.

In this study, we apply PC method to define the optimal window size. The basic idea is that we attempt to find the largest window size in which $c_{0}$ proportion of the total information can be explained by the first $k$ PCs and $c_{0}$ and $k$ are predefined criteria. We define this largest window size as the optimal window size. Start with a window $w_{l}^{b}$ with $l=s=k+1$, so that at least the window length is longer than the number of the important PCs.

Let $\sum_{g}^{b}$ denote the sample variance-covariance matrix of genotypic numerical codes in window $w_{l}^{b}$ and $\lambda_{j}^{b}$ denote the $j^{\text {th }}$ largest eigenvalue of $\sum_{g}^{b}$ Thus, in window $w_{l}^{b}$, the total variance in the original dataset explained by the $j^{\text {th }}$ PC is $\lambda_{j}^{b} /\left(\lambda_{1}^{b}+\lambda_{2}^{b}+\cdots+\lambda_{l}^{b}\right)$. Let $C=\left(\lambda_{1}^{b}+\cdots+\lambda_{k}^{b}\right) /\left(\lambda_{1}^{b}+\lambda_{2}^{b}+\cdots+\lambda_{l}^{b}\right)$ as the proportion of the total variability explained by the first $k$ PCs. Our main idea of choosing the optimal window size of each sliding window is to find the largest window size in which $c_{0}$ proportion of the total variability can be explained by the first $k$ PCs among a set of windows $\Omega^{b}$.

\section{Bisection method for searching the optimal window size and computational consideration}

Using the exhaustive searching method may be computational demanding for determining the optimal window size. We propose to use bisection method. Let $s$ and $\Gamma$ denote the predefined smallest and largest window sizes among a set of windows $\Omega^{b}$, where $\mathrm{b}$ is the starting SNP of the set of windows.

By adapting bisection method, the searching procedure for the optimal window size in $\Omega^{b}$ includes following steps:

Step 1: Let $l$ be the middle point of $s$ and $\Gamma$, that is, $l=$ $[(s=\Gamma) 2 /]$, where [a] is the largest integer that is less than or equal to $\mathrm{a}$.

Step 2: Conduct PC analysis within the window $w_{l}^{b}$, where a window begins at SNP $b$ and has a size $l$.

Step 3: Calculate $C$ (the proportion of the total variability explained by the first $k$ PCs) for the window $w_{l}^{b}$. If $C>c_{0}$, we let $s=l$, that is, we update the smallest window size $s$. Otherwise, we let $\Gamma=l$, that is, we update the largest window size $\Gamma$.

Step 4: Repeat Step 1 to Step 3 until $\Gamma-s \leq 1$.

In the window $w_{\Gamma}^{b}$, if the proportion of the total variability explained by the first $k$ PCs is greater than $c_{0}$, the optimal window size will be $\Gamma$; otherwise, the optimal window size will be $s$.

Until now, we have not mentioned how to choose the starting SNP $b$. Of course for the first window, $b=1$. To choose $b$ for other windows, the following three methods are typically used. For the $i^{\text {th }}(i>1)$ window, choose 1) $b=i$; 2) $b=n_{i}$, where $n_{i}$ is the middle SNP of the $(i-1)^{\text {th }}$ window; 3) $b=m_{i}+1$, where $m_{i}$ is the last SNP of the $(i-1)^{\text {th }}$ window. In this article, we use the first method to choose the starting SNP $b$.

By using bisection method, our proposed variable length sliding-window method is computationally efficient. Consider a set of windows $\Omega^{b}$ with the smallest window size $s$, largest window size $\Gamma$, and starting SNP $b$. The computational complexity to find the optimal window size in $\Omega^{b}$ using the bisection algorithm is $\Gamma^{3} \log _{2}(\Gamma-s)$. If we have N SNPs in total, the computational complexity to find all the optimal window sizes is $N \Gamma^{3} \log _{2}(\Gamma-s)$. In this article, we use $\Gamma=35$ and $s=4$. Suppose $N=500,000$ 
in a genome-wide association study. Then, $N \Gamma^{3} \log _{2}(\Gamma-s)$ $<N^{2}$. As pointed out by one of the reviewers, HAPLOVIEW program may be used to find beginning and end of a window. Using HAPLOVIEW, $N^{2}$ of pair-wise $r^{2}$ need to be calculated. To calculate $r^{2}$, we need to estimate haplotype frequencies. Theoretically, our proposed method should be computationally more efficient than HAPLOVIEW. In fact, we have done a preliminary simulation study. The results show that the computation time of our proposed method is about a hundred times faster than HAPLOVIEW.

\section{Score test}

After we find the optimal window size for each sliding window, we use the score test statistic based on a logistic model [5] to test for association within each sliding window. Consider $w_{l}^{b}$, a window beginning at SNP $b$ with an optimal window size $l$. Take $b=1$ as an example for windows that start at the first SNP. Let $x_{i}=\left(x_{i 1}^{*}, x_{i 2}^{*}, \cdots, x_{i k}^{*}\right)^{T}$ denote its first $k$ PCs of the $i^{\text {th }}$ individual, where $i=1,2, \ldots, M$. Suppose that the $k$ PCs follow a logistic model, then, the score test statistic is given by $T^{2}=U^{\prime} V^{-1} U$, where $U=\sum_{i=1}^{M}\left(y_{i}-\bar{\gamma}\right)\left(x_{i}-\bar{x}\right)$, $\operatorname{var}(\gamma)=\frac{1}{M} \sum_{i=1}^{M}\left(y_{i}-\bar{\gamma}\right)^{2}, \operatorname{var}(\gamma)=\frac{1}{M} \sum_{i=1}^{M}\left(y_{i}-\bar{\gamma}\right)^{2}$, and $M$ is the sample size. The statistic $T^{2}$ asymptotically follows a $\chi^{2}$ distribution with $k$ degrees of freedom. We select significant windows after adjusting for multiple testing using a Bonferroni correction.

\section{Result}

We applied the proposed approach to GAW16 Problem 1. In our application, we set $s=4 ; \Gamma=35 ; c_{0}=90 \%$ and $k=3$. Originally the dataset contained genotypes at 545,080 SNPs on chromosomes 1 to 22 . In our analysis we ended up with 531,501 windows. The size of the windows varied from 4 to 29 SNPs, with the median window size of 7 (see Table 1 for the distribution of the window sizes). After Bonferroni correction, we found 1,155 significant

Table I: The distribution of the window sizes based on 531,50 I windows

\begin{tabular}{lc}
\hline Window size & Percentage of total windows \\
\hline 4 & 18.32 \\
5 & 11.92 \\
6 & 11.69 \\
7 & 11.57 \\
8 & 8.94 \\
9 & 7.34 \\
10 & 6.34 \\
11 & 4.67 \\
12 & 3.73 \\
$13-29$ & 15.48 \\
\hline
\end{tabular}

windows. Due to the strong LD among SNPs, many of the significant windows overlapped with the nearby windows. In order to report the result thoroughly, we combined the significant windows with all overlapped windows as one larger window. Thus, we end up with 76 significant larger windows. Due to the limited pages, in Table 2 we only report the top 30 windows after the combination. The order of the windows is according to their most significant sub-windows (the original window before combinations). Our result matches most of the genes reported in recent studies [6-11] and also identify more rheumatoid arthritis (RA) susceptibility genes for follow-up studies.

\section{Discussion}

As the most exhaustive searching engine in genome-wide association studies, sliding-window approaches are receiving more and more attention recently. Based on the variable sized sliding-window frame, we adapt the optimal window size to the local LD pattern by employing the PC approach. We applied this novel sliding-window approach to the GAW16 RA data and successfully validated nine genes that have been reported by recent studies and also identified new candidate genes for follow-up studies.

Our approach has several advantages. It provides a stable method to choose the window size with the maximum information extraction and it automatically balances degrees of freedom and number of tests, which results in higher power to detect association. It is flexible enough to conduct different association tests within the windows. The method is computational efficient when applied to largescale data compared with other variable sized slidingwindow methods. It requires only genotype data so there is no need to go through any computationally intensive phasing program to account for uncertain haplotype phases.

Further efforts are needed to improve the proposed method, such as determining the optimal $c_{0}$ (the proportion of the total variability explained by the top k PCs) and the initial window lengths in the bisection method.

\section{Conclusion}

In this study, we applied our novel genome-wide PC slidingwindow approach to detect the association between SNP windows and disease status using GAW16 Problem 1 RA dataset. We validated nine genes which have been identified to be responsible for RA in the literature and discovered more genes and non-gene regions for follow-up studies.

\section{List of abbreviations used}

GAW: Genetic Analysis Workshop; LD: Linkage disequilibrium; NARAC: North American Rheumatoid Arthritis 
Table 2: Genetic and physical map locations of window region identified using PC-sliding-window analysis based on the Bonferroni correction

\begin{tabular}{|c|c|c|c|c|}
\hline Window ID & Chr ${ }^{a}$ & Physical location & Genes $^{\text {b }}$ & CRASG $^{\mathrm{C}}$ \\
\hline I & 6 & $300|4670,33| 87 \mid 44$ & TNF, HLA-A HLA-B, HLA-C & TNF, HLA-A HLA-B, HLA-C \\
\hline 2 & 1 & 792429,1101089 & AGRN, CIorfI59, ISGI5, SAMDII & \\
\hline 3 & 2 & I72768404, I72807000 & $D L X 1, D L X 2$ & STAT4, ITGAV \\
\hline 4 & 12 & 46666298,46718200 & COL2AI, LOC728I8I LOC728II4 & \\
\hline 5 & 13 & $113656958,11386 \mid 908$ & FAM70B, RASA3 & \\
\hline 6 & 7 & $|54| 3320|| 5424|| 60$, & PAXIPI, LOC20278I & \\
\hline 7 & 17 & 68283979,68361160 & SLC39AII & \\
\hline 8 & 2 & 98261543,98370780 & VWA3B & ILIB \\
\hline 9 & 13 & 49336428,49340230 & KPNA3 & \\
\hline 10 & I & 2243956, 3359357 & ARHGEFI6, PRDM I6 & \\
\hline II & 17 & 66647226,66750860 & Intergenic 17q24 & \\
\hline 12 & 16 & 67482002,67660490 & TMC07, FLJI233I & \\
\hline 13 & 18 & $75300466,753|4| 40$ & NFATCI & \\
\hline 14 & 13 & 74883232,74941310 & TBCID4 & \\
\hline 15 & 9 & $|232| \mid 883,123248000$ & CRB2, MIRN60I, DENNDIA & TRAFI/C5 \\
\hline 16 & 20 & 57796484,57832810 & & \\
\hline 17 & I & 15181683,151846600 & ADAM I5, EFNA4 & \\
\hline 18 & 20 & $35438689,3550 \mid 280$ & SRC, RPL7AL4 & \\
\hline 19 & 22 & 28164734,28237820 & RFPLIS, RFPLI, NEFH & \\
\hline 20 & 11 & $64593946,6466 \mid 480$ & SAC3DI, NAALADLI CDCA5, ZFPLI, ZHIT2 & \\
\hline 21 & 11 & 45207308,45314100 & SYTI3, FLJ4 I 423 & \\
\hline 22 & 8 & 20327035,20435200 & & \\
\hline 23 & 5 & $|376| 4229,137825000$ & GFRA3, CDC25C, FAM53C, JMJDIB & \\
\hline 24 & 7 & 129525353,129580000 & CPA2, CPA4 & \\
\hline 25 & 3 & 134954925,134966000 & $T F, S R P R B$ & \\
\hline 26 & 9 & 104811123,104815000 & ABCAI & \\
\hline 27 & 12 & 6924169,6932652 & ATNI, CL2orf57, PTPN6 & \\
\hline 28 & 10 & |053|I8I, I054284| & $S H 3 P X D 2 A$ & \\
\hline 29 & 11 & 3335218,3524620 & ZNFI95, OR7ZI2p & \\
\hline 30 & 19 & 19106771,19154190 & TMEMI6AI, MEF2B & \\
\hline
\end{tabular}

${ }^{\mathrm{a} C h r}$, chromosome.

${ }^{b}$ We found the significant genes using the NCBI dbSNP database http://www.ncbi.nlm.nih.gov/sites/entrez?db=snp.

'The confirmed RA susceptibility genes (CRASG) are shown in the last column if they are within or near our significant region.

Consortium; PC: Principal components; RA: Rheumatoid arthritis; SNP: Single-nucleotide polymorphism.

\section{Competing interests}

The authors declare that they have no competing interests.

\section{Authors' contributions}

QS participated in the design of the study and contributed to the manuscript preparation. RT performed the statistical analysis and wrote the draft of the manuscript. SZ contributed to the design of the study and to the manuscript preparation. All authors read and approved the final manuscript.

\section{Acknowledgements}

The Genetic Analysis Workshops are supported by NIH grant ROI GM031575 from the National Institute of General Medical Sciences.

This work was supported by $\mathrm{NIH}$ grants ROI GM069940 and the Overseas-Returned Scholars Foundation of Department of Education of Heilongjiang Province (I I52HZOI).
This article has been published as part of BMC Proceedings Volume 3 Supplement 7, 2009: Genetic Analysis Workshop 16. The full contents of the supplement are available online at http://www.biomedcentral.com/ $1753-656 \mid / 3$ ? issue $=$ S7.

\section{References}

I. Yang HC, Lin CY and Fann CSJ: A sliding-window weighted linkage disequilibrium test. Genet Epidemiol 2006, 30:53I-545.

2. Li $Y$, Sung $W$ and Liu JJ: Association mapping via regularized regression analysis of single-nucleotide-polymorphism haplotypes in variable-sized sliding windows. Am J Hum Genet 2007, 80:705-715.

3. Huang $\mathrm{BE}, \mathrm{Amos} \mathrm{Cl}$ and Lin DY: Detecting haplotype effects in genomewide association studies. Genet Epidemiol 2007, 3I:803-8I2.

4. Sha Q, Dong J, Jiang R and Zhang S: Test of association between quantitative traits and haplotypes in a reduced-dimensional space. Ann Hum Genet 2005, 69:715-732.

5. Chapman JM, Cooper JD, Todd JA and Clayton DG: Detecting disease associations due to linkage disequilibrium using haplotype tags: a class of tests and the determinants of statistical power. Hum Hered 2003, 56:|8-3|.

6. Begovich AB, Carlton VE, Honigberg LA, Schrodi SJ, Chokkalingam AP, Alexander HC, Ardlie KG, Huang Q, Smith AM, Spoerke JM, Conn MT, Chang M, Chang SY, Saiki RK, Catanese J], Leong DU, Garcia VE, McAllister LB, Jeffery DA, Lee AT, Batliwalla F, Remmers E, Criswell LA, Seldin MF, Kastner DL, Amos Cl, Sninsky I] and Gregersen PK: A missense single-nucleotide polymorphism in a gene encoding a protein tyrosine phosphatase 
(PTPN22) is associated with rheumatoid arthritis. Am J Hum Genet 2004, 75:330-337.

7. Thomson W, Barton A, Ke X, Eyre S, Hinks A, Bowes J, Donn R, Symmons D, Hider S, Bruce IN, Wellcome Trust Case Control Consortium, Wilson AG, Marinou I, Morgan A, Emery P, YEAR Consortium, Carter A, Steer S, Hocking L, Reid DM, Wordsworth P, Harrison P, Strachan D and Worthington J: Rheumatoid arthritis association at 6q23. Nat Genet 2007, 39:| |43|-| 433.

8. Remmers EF, Plenge RM, Lee AT, Graham RR, Hom G, Behrens TW, de Bakker PI, Le JM, Lee HS, Batliwalla F, Li W, Masters SL, Booty MG, Carulli JP, Padyukov L, Alfredsson L, Klareskog L, Chen WV, Amos Cl, Criswell LA, Seldin MF, Kastner DL and Gregersen PK: STAT4 and the risk of rheumatoid arthritis and systemic lupus erythematosus. N Engl J Med 2007, 357:977-986.

9. Plenge RM, Seielstad M, Padyukov L, Lee AT, Remmers EF, Ding B, Liew A, Khalili H, Chandrasekaran A, Davies LR, Li W, Tan AK, Bonnard C, Ong RT, Thalamuthu A, Pettersson S, Liu C, Tian C, Chen WV, Carulli JP, Beckman EM, Altshuler D, Alfredsson L Criswell LA, Amos Cl, Seldin MF, Kastner DL, Klareskog L and Gregersen PK: TRAFI-C5 as a risk locus for rheumatoid arthritis-a genomewide study. N Engl J Med 2007, 357:1199-1209.

10. Kurreeman FA, Padyukov L, Marques RB, Schrodi SJ, Seddighzadeh M, Stoeken-Rijsbergen G, Helm-van Mil van der AH, Allaart CF, Verduyn W, Houwing-Duistermaat J, Alfredsson L, Begovich AB, Klareskog L, Huizinga TW and Toes RE: A candidate gene approach identifies the TRAFI/C5 region as a risk factor for rheumatoid arthritis. PLoS Med 2007, 4:e278.

II. Jacq L, Garnier S, Dieudé P, Michou L, Pierlot C, Migliorini P, Balsa A Westhovens R, Barrera P, Alves H, Vaz C, Fernandes M, PascualSalcedo D, Bombardieri S, Dequeker J, Radstake TR, Van Riel P, Putte van de L, Lopes-Vaz A, Glikmans E, Barbet S, Lasbleiz S, Lemaire I, Quillet P, Hilliquin P, Teixeira VH, Petit-Teixeira E, Mbarek H, Prum B, Bardin T, Cornélis F and European Consortium on Rheumatoid Arthritis Families: The ITGAV rs37389/9-C allele is associated with rheumatoid arthritis in the European Caucasian population: a family-based study. Arthritis Res Ther 2007, 9:R63.
Publish with BioMed Central and every scientist can read your work free of charge

"BioMed Central will be the most significant development for disseminating the results of biomedical research in our lifetime. "

Sir Paul Nurse, Cancer Research UK

Your research papers will be:

- available free of charge to the entire biomedical community

- peer reviewed and published immediately upon acceptance

- cited in PubMed and archived on PubMed Central

- yours - you keep the copyright
BioMedcentral 